\title{
Carrying Angle variations between dominant and non-dominant Limb in Jammu region of North India
}

\author{
Authors \\ Imtiyaz Ahmad Beigh", Tanveer Ahmed Bhat ${ }^{2 *}$, Sayar Ahmad Mantoo ${ }^{3}$, \\ Suhail Ahmad Bhat ${ }^{4}$, John Mohammad ${ }^{5}$, Zubair Ahmad lone ${ }^{6}$ \\ ${ }^{1,2,3}$ SR, Orthopedics, SGRRIM \& HS, Dehradun, ${ }^{4}$ Research Fellow, Pondicherry University \\ ${ }^{5} \mathrm{SR}$, Orthopedics, GMC, Jammu, ${ }^{6} \mathrm{PGY} 3, \mathrm{GMC}$, Jammu \\ *Corresponding Author \\ Tanveer Ahmed Bhat
}

\begin{abstract}
Background: The angle formed by median axis of the arm with fully extended and supinated forearm is known by the name of carrying angle(CA). Carrying angle is important as it allows forearms to clear hips in swinging movements while walking and while carrying objects.

Objective: The aim of this study was to measure carrying angle of a sample population in the age group 18-30 years manually allowing comparison between measurements of dominant and non dominant limb and document any differences thereof.

Material and Methods: Carrying angle measurements were carried out in 300 individuals in the age group 18-30 years using goniometer. Measurements were done in both the upper limbs with limb kept in full extension at elbow and supination at forearm during measurement.

Results: After documentation of measurements our results showed significant difference in carrying angles of dominant and non dominant limbs. Our study also revealed variation of carrying angle between females and males with females having values greater in both dominant and non-dominant limbs as compared to males.

Conclusion: Our study emphasized on the fact that carrying angle measurement was more in case of non dominant limb as compared to dominant limb and was more in case females than males.

Keywords: CA, Post-Pubertal Age, Clinical Methods, Elbow Prostheses.
\end{abstract}

\section{Introduction}

The carrying angle refers to the angle formed by long axis of the arm and forearm in the frontal plane with the elbow in full extension and the forearm in supination. As a result of this position, the forearm does not lie in a straight line with the arm, deviating laterally with respect to the arm axis forming the carrying angle. The average value of carrying angle is $12.5 \pm 0.57$ degrees in males and $15.26 \pm 0.45$ degrees in females. This apparent variability in gender may be because of joint laxity which is particularly higher in females permitting a greater degree of elbow extension and the resultant carrying angle therefore ${ }^{1}$ In addition to this significant Variations in carrying angle among different age groups, gender and race has also been reported in literature with Females having higher values than males in age group of 18-30 years. 
The carrying angle is affected to some extent by various pathological conditions of lateral and medial epicondyles. Thus the evaluation of carrying angle value and its pathologic variations are of paramount significance to identify the deformities at elbow and in the diagnosis of various pathological conditions of the lateral and medial epicondyles. ${ }^{1,2}$

With Increase in value of the carrying angle there is possibility of elbow instability and pain during exercise or in throwing activities of sports, predisposing to increased risk of elbow dislocation and distal humerus fractures while falling on an outstretched hand. ${ }^{4}$ The rate of increment in carrying angle for boys and girls is 0.42 and 0.60 per year, respectively. ${ }^{4}$ In normal physiological conditions carrying angle measurement varies according to age ${ }^{5}$, gender ${ }^{6}$, hyperextension of the elbow ${ }^{7}$, dominant upper $\operatorname{limb}^{8}$, anthropometric characteristics such as height and intertrochanteric distance ${ }^{9}$ and this can be measured by simple clinical and radiographic technique $^{10}$.

This study was conducted to compare data obtained by measuring CA by manual method between dominant and non dominant limb in the age group $18-30$ years.

\section{Material and Methods}

The study was conducted on 250 subjectsaged between 18 - 30 years at government medical college Jammu from May 2017 to May 2018. Subjects selected after properly obtaining the consent directly from the subject. Both male as well as female subjects participated in our study. those subjects with congenital deformities of one or both the upper extremeties, history of any fractures and surgeries around the elbow joint, endocrine disorders affecting skeletal systems and bone disorders due to the Vitamin deficiencies were excluded from the study.

\section{Materials}

The materials Used were Measuring Scale, measuring Tape, marker pen, clinical goniometer $180^{\circ}$.

\section{Methods}

While taking into account all the inclusion and exclusion criteria subjects were selected and initial assessment was done. the initial clinical assessment involved a thorough clinical examination of both the upper extremities including the elbow joints. After taking informed consent the carrying angle of both the upper limbs in each subjects was clinically measured by means of goniometer. During the measurement the elbow was kept in complete extension. For each limb in every subject measurement was taken three times with the final measurement being the average of three readings. Readings were obtained with the help of goniometer. An improvised instrument goniometer was used for measuring the carrying angle. The fixed arm of goniometer was placed on the median axis of the upper arm, the movable arm adjusted as to lie on the median axis of forearm and the angle read on the goniometer as shown in figure 1 . The bicipital groove, biceps brachii tendon at its insertion and Palmaris longus tendon at the wrist were palpated and were marked as anatomical landmarks to For demarcation of median axes of the arm and the forearm The bicipital groove, biceps brachii tendon at its insertion and Palmaris longus tendon at the wrist were palpated and were marked as anatomical landmarks Carrying angle was measured on both sides and difference documented.

\section{Statistical analysis and Results}

The continuous parameters were expressed as the mean with standard deviation using Independent Student's t-test. All statistical analysis were carried out at $5 \%$ level of significance and p-value $<0.05$ was considered as significant. 


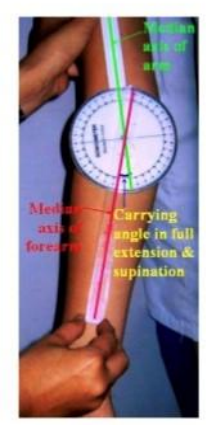

Fig. 1. Clinical image showing method of carrying angle measurement

This study included 250 volunteers, 141 males and 109 females. In this study right arm dominance was seen in 230 (92\%) subjects with left arm dominance found in 20(8\%) subjects. The carrying in females was found to be more as compared to males in dominant as well as non dominant limbs (table 1). In almost all of the subjects carrying angle of dominant right limb was greater than the non dominant left limb irrespective of gender difference (table 2). In case of males, the right carrying angle was $12.18 \pm 1.38$ and the left carrying angle was $10.17 \pm 1.32$ $(P<.001)$ (Table 3). In case of females, right carrying angle was $14.53 \pm 1.92$ and left carrying angle was $13.43 \pm 1.62(P<0.001)$ (Table 3$)$.

\section{Discussion}

Our study suggested that natural forces do act on elbow to modify carrying angle as a result of which the Carrying angle was found to be greater in the dominant limb than the non-dominant limb irrespective of gender difference. The stress imposed over the dominant limb may result in developmental changes contributing to changes in the carrying angle. In addition to this aging and racial influences may add further to the variability of this parameter. Knowledge of the measurement of the carrying angle and its variations is important while evaluating traumatic elbow injuries in childhood and adolescence and other elbow disorders that requiring reconstruction or replacement surgeries. Sharma k Mansur et al in their study Variation of Carrying Angle With Age, Sex, Height and Special Reference to Side have also found that the carrying angle of dominant limb is more than the non-dominant one ${ }^{2}$.CA measurements and its behavior with respect to different genders and the sides of limb is important for preoperative evaluation and surgical management of traumatic elbow injuries. It also has an important role in the correction of congenital deformities influencing the elbow joint, treatment of epicondylar diseases It does play a significant role in designing of elbow prosthesis in case of arthroplasties. For a better design of resurfacing and semi constrained elbow replacement implants, detailed evaluation and knowledge of elbow joint anatomical features and CA parameters are of paramount importance. Thus before advancing with surgeries for correction of deformities of elbow joint whether traumatic or atraumatic and replacement surgeries a surgeon should be well aware of carrying angle parameters and its changing patterns with respect to limb dominance or gender difference.

Table-1: Shows values in the range mean and standard deviation of carrying angle of males and females of Jammu population.

\begin{tabular}{|l|l|c|l|}
\hline & Male $(\mathrm{n}=141)$ & Female $(\mathrm{n}=109)$ & $\mathrm{P}$ value \\
\hline Right arm & $12.18 \pm 1.38$ & $14.53 \pm 1.92$ & $<0.001$ \\
\hline Left arm & $10.17 \pm 1.32$ & $13.43 \pm 1.62$ & $<0.001$ \\
\hline
\end{tabular}

Table-2: Shows pooled mean and standard deviation of carrying angle of dominant and non dominant limb.

\begin{tabular}{|l|l|c|c|}
\hline Total volunteers & Right arm & Left arm & P value \\
\hline 250 & $13.28 \pm 1.42$ & $12.26 \pm 1.54$ & $<0.001$ \\
\hline
\end{tabular}

Table-3: Comparison in values of carrying angle of males and females of Jammu population

\begin{tabular}{|l|c|c|c|}
\hline & Right arm & Left arm & $P$ value \\
\hline Males $(\mathrm{n}=141)$ & $12.18 \pm 1.38$ & $10.17 \pm 1.32$ & $<0.001$ \\
\hline females $(\mathrm{n}=109)$ & $14.53 \pm 1.92$ & $13.43 \pm 1.62$ & $<0.001$ \\
\hline
\end{tabular}

Chang Hung Chu et al, ${ }^{11}$ concluded that an increased carrying angle of the elbow appeared to be an independent risk factor for the non-traumarelated ulnar neuropathy. Knowledge of carrying angle of the elbow and its variability is important when evaluating traumatic elbow injuries in childhood and in adolescence and other elbow 
disorders that require reconstruction ${ }^{12}$ or arthroplasties (surface and semi- constrained) ${ }^{13}$.

\section{Conclusion}

Our study concluded that the Carrying angle is greater in the dominant limb than the nondominant limb of both genders. The carrying angle measurement of female sex is slightly higher than males in both dominant as well as non-dominant limb. While dealing with and treatment of traumatic elbow injuries in childhood and in adolescence and other elbow disorders that require reconstruction or arthroplasties the normal parametric values of carrying angle should be duly considered.

\section{References}

1. Vichard Lim, Natasha Ashley Jacob. The carrying angle of elbow, An Anthropometric Study on the Carrying Angle of Elbow among Young Adults of Various Ethinicities in Malaysia, NJIRM 2014; 5:2230 - 9969.

2. Sharma K, Mansur DI, Khanal K, Haque MK. Variation of Carrying Angle With Age, Sex, Height and Special Reference to Side, Kathmandu Univ Med J 2013;44:315-318.

3. Chein-Wei Chang, Yi-Chian Wang, Chang-Hung Chu. Increased Carrying Angle is a Risk Factor for Nontraumatic Ulnar Neuropathy at the Elbow, The Association of Bone and Joint Surgeons 2008; 466:2190-2195.

4. Jyothinath Kothapalli1, Pradeepkumar H. Murudkar1, Lalitha Devi. The carrying angle of elbow- a correlative and comparative study. Int J Cur Res Rev, 2013/ Vol 05 (07)

5. Beals RK. The normal carrying angle of the elbow. A radiographic study of 422 patients. Clin Orthop Relat Res.1976; 119:194-6.

6. Zampagni ML, Casino D, Zaffagnini S, Visani AA, Marcacci M. Estimating the elbow carrying angle with an electrogoniometer: acquisition of data and reliability of measurements. Orthopedics. 2008;31(4):370.

7. Golden DW, Jhee JT, Gilpin SP, Sawyer JR. Elbow range of motion and clinical carrying angle in a healthy pediatric population. J Pediatr Orthop B.2007; 16:144-9.

8. Tükenmez $\mathrm{M}$, Demirel $\mathrm{H}$, Perçin $\mathrm{S}$, Tezeren G. Measurement of the carrying angle of the elbow in 2,000 children at ages six and fourteen years. Acta Orthop Traumatol Turc.2004;38:274-6.

9. Paraskevas G, Papadopoulos A, Papaziogas B, Spanidou S, Argiriadou H, Gigis J. Study of the carrying angle of the human elbow joint in full extension: a morphometric analysis.SurgRadiol Anat. 2004;26:19-23.

10. Balasubramanian P, Madhuri V, Muliyil J. Carrying angle in children: a normative study. J Pediatr Orthop B. 2006; 15:37-40.

11. Chein-WC, Yi-Chian W. and Chang-HC. Increased Carrying Angle is a Risk Factor for Nontraumatic Ulnar Neuropathy at the Elbow. Clin Orthop Relat Res. 2008,;466: 2190-2195.

12. Zampagni ML, Casino D, Martelli S, Visani A, Marcacci M. A protocol for clinical evaluation of the carrying angle of the elbow by anatomic landmarks. J Shoulder Elbow Surg. 2008;17:106-12.

13. An KN, Morrey BF, Chao EY. Carrying angle of the human elbow joint. J Orthop Res. 1984;1:369-78. 